\title{
Research Capability Measures for Processes with Multiple Characteristics
}

\author{
K. S. Chen ${ }^{1}$, W. L. Pearn ${ }^{2, *, \dagger}$ and P. C. $\operatorname{Lin}^{3}$ \\ ${ }^{1}$ Department of Industrial Engineering and Management, National Chin-Yi Institute of Technology, Taichung, Taiwan \\ ${ }^{2}$ Department of Industrial Engineering and Management, National Chiao Tung University, Taiwan \\ ${ }^{3}$ Center of General Education, National Chin-Yi Institute of Technology, Taiwan
}

Process capability indices, such as $C_{p}, C_{a}$, and $C_{p k}$, have been widely used in the manufacturing industry providing numerical measures on process precision, process accuracy, and process performance. Capability measures for processes with a single characteristic have been investigated extensively. However, capability measures for processes with multiple characteristics are comparatively neglected. In this paper, we consider a generalization of the yield index $S_{p k}$ proposed by Boyles, for processes with multiple characteristics. We establish a relationship between the generalization and the process yield. We also develop a control chart based on the proposed generalization, which displays all the characteristic measures in one single chart. Using the chart, the engineers can effectively monitor and control the performance of all process characteristics simultaneously. Copyright (c) 2003 John Wiley \& Sons, Ltd.

KEY WORDS: capability zone; MCPCA chart; multiple characteristics; process capability index; process yield index

\section{INTRODUCTION}

$\mathrm{P}$ rocess capability indices, establishing the relationship between the actual process performance and the manufacturing specifications, have been the focus of recent research in quality assurance and capability analysis. Those capability indices quantifying process potential and process performance are essential to any successful quality improvement activities and quality program implementation. Some basic capability indices that have been widely used in the manufacturing industry include $C_{p}, C_{a}$, and $C_{p k}$, explicitly defined as follows ${ }^{1-3}$ :

$$
\begin{aligned}
C_{p} & =\frac{\mathrm{USL}-\mathrm{LSL}}{6 \sigma} \\
C_{a} & =1-\frac{|\mu-m|}{d} \\
C_{p k} & =\min \left\{\frac{\mathrm{USL}-\mu}{3 \sigma}, \frac{\mu-\mathrm{LSL}}{3 \sigma}\right\}
\end{aligned}
$$

\footnotetext{
*Correspondence to: W. L. Pearn, Department of Industrial Engineering and Management, National Chiao Tung University, 1001 TA Hsueh Road, Hsinchu, 30050 Taiwan.

†E-mail: roller@cc.nctu.edu.tw 
where USL and LSL are the upper and the lower specification limits, respectively, $\mu$ is the process mean, $\sigma$ is the process standard deviation, $m=(\mathrm{USL}+\mathrm{LSL}) / 2$ is the mid-point of the specification interval, and $d=(\mathrm{USL}-\mathrm{LSL}) / 2$ is half the length of the specification interval.

The index $C_{p}$ measures the overall process variation relative to the specification tolerance, therefore it only reflects process potential (or process precision). The index $C_{a}$ measures the degree of process centering, which alerts the user if the process mean deviates from its target value. Therefore, the index $C_{a}$ only reflects process accuracy. The index $C_{p k}$ takes into account the magnitude of process variation as well as the degree of process centering, which measures process performance based on yield (proportion of conformities). For a normally distributed process with a fixed value of $C_{p k}$, the bounds on process yield, \% Yield, are given by $2 \Phi\left(3 C_{p k}\right)-1 \leq \%$ Yield $<\Phi\left(3 C_{p k}\right)$, where $\Phi(\cdot)$ is the cumulative distribution function of $N(0,1)$, the standard normal distribution. For example, if $C_{p k}=1.00$, then it guarantees that the \%Yield will be no less than $99.73 \%$, or no greater than $2700 \mathrm{ppm}$ (parts per million) of non-conformities. We note that the index $C_{p k}$ only provides an approximate rather than an exact measure of the process yield. To obtain an exact measure, Boyles ${ }^{4}$ considered a yield index, referred to as $S_{p k}$, for normally distributed processes. The index $S_{p k}$ is defined as:

$$
S_{p k}=\frac{1}{3} \Phi^{-1}\left\{\frac{1}{2} \Phi\left(\frac{\mathrm{USL}-\mu}{\sigma}\right)+\frac{1}{2} \Phi\left(\frac{\mu-\mathrm{LSL}}{\sigma}\right)\right\}
$$

where $\Phi^{-1}$ is the inverse function of $\Phi$. For a process with $S_{p k}=c$, we can obtain \% Yield $=2 \Phi(3 c)-1$. Obviously, there is a one-to-one relationship between $S_{p k}$ and the process yield. Thus, the yield index $S_{p k}$ provides an exact measure of the process yield. For normally distributed processes, the number of nonconformities corresponding to a capable process with $S_{p k}=1.00$ is $2700 \mathrm{ppm}$, a satisfactory process with $S_{p k}=1.33$ is $63 \mathrm{ppm}$, an excellent process with $S_{p k}=1.67$ is $0.6 \mathrm{ppm}$, and a super process with $S_{p k}=2.00$ is $0.002 \mathrm{ppm}$.

\section{CAPABILITY MEASURE FOR MULTIPLE CHARACTERISTICS}

Capability measures for processes with a single characteristic have been investigated extensively, see e.g. Kane $^{1}$, Pearn et al. ${ }^{2,3}$, Boyles ${ }^{4}$, Chan et al. ${ }^{5}$, Choi and Owen ${ }^{6}$, Kotz and Johnson ${ }^{7}$, Vännman ${ }^{8}$, Deleryd and Vännman ${ }^{9}$ and Pearn and $\operatorname{Lin}^{10}$. However, capability measures for processes with multiple characteristics is comparatively neglected. For processes with multiple characteristics, Bothe ${ }^{11}$ considered a simple measure by taking the minimum measure of each single characteristic. For example, consider a $v$-characteristic process with $v$ yield measures (percentage of conformities) $P_{1}, P_{2}, \ldots$, and $P_{v}$. The overall process yield is measured as $P=\min \left\{P_{1}, P_{2}, \ldots, P_{\nu}\right\}$. We note that this approach does not reflect the real situation accurately. Suppose the process has five characteristics $(v=5)$, with equal characteristic yield measures $P_{1}=P_{2}=P_{3}=P_{4}=P_{5}=99.73 \%$. Using the approach considered by Bothe ${ }^{11}$, the overall process yield is calculated as $P=\min \left\{P_{1}, P_{2}, P_{3}, P_{4}, P_{5}\right\}=99.73 \%$ (or $2700 \mathrm{ppm}$ of non-conformities). Assuming that the five characteristics are mutually independent, then the actual overall process yield should be calculated as $P=P_{1} \times P_{2} \times \cdots \times P_{5}=98.66 \%$ (or $134273 \mathrm{ppm}$ of non-conformities), which is significantly less than that calculated by Bothe ${ }^{11}$.

To overcome the problem, we propose the following overall capability index, referred to as $S_{p k}^{\mathrm{T}}$ :

$$
S_{p k}^{\mathrm{T}}=\frac{1}{3} \Phi^{-1}\left\{\left[\prod_{j=1}^{v}\left(2 \Phi\left(3 S_{p k j}\right)-1\right)+1\right] / 2\right\}
$$

where $S_{p k j}$ denotes the $S_{p k}$ value of the $j$ th characteristic for $j=1,2, \ldots, v$, and $v$ is the number of characteristics. The new index, $S_{p k}^{\mathrm{T}}$, may be viewed as a generalization of the single characteristic yield index, $S_{p k}$, considered by Boyles ${ }^{4}$. 
Table I. Various $S_{p k}^{\mathrm{T}}$ values and the corresponding process yield

\begin{tabular}{cc}
\hline$S_{p k}^{\mathrm{T}}$ & Process yield \\
\hline 1.00 & 0.997300204 \\
1.24 & 0.999800777 \\
1.33 & 0.999933927 \\
1.50 & 0.999993205 \\
1.67 & 0.999999456 \\
2.00 & 0.999999998 \\
\hline
\end{tabular}

Given $S_{p k}^{\mathrm{T}}=c$, we have

$$
\frac{1}{3} \Phi^{-1}\left\{\left[\prod_{j=1}^{v}\left(2 \Phi\left(3 S_{p k j}\right)-1\right)+1\right] / 2\right\}=c
$$

Hence,

$$
\prod_{j=1}^{v}\left[2 \Phi\left(3 S_{p k j}\right)-1\right]=2 \Phi(3 c)-1
$$

A one-to-one correspondence relationship between the index $S_{p k}^{\mathrm{T}}$ and the overall process yield $P$ can be established as:

$$
P=\prod_{j=1}^{v} P_{j}=\prod_{j=1}^{v}\left[2 \Phi\left(3 S_{p k j}\right)-1\right]=2 \Phi\left(3 S_{p k}^{\mathrm{T}}\right)-1 .
$$

Hence, the new index $S_{p k}^{\mathrm{T}}$ provides an exact measure of the overall process yield. For example, if $S_{p k}^{\mathrm{T}}=1.00$, then the entire process yield would be exactly $99.73 \%$. Table I displays various commonly used capability requirements and the corresponding overall process yield. For a process with $v$ characteristics, if the requirement for the overall process capability is $S_{p k}^{\mathrm{T}} \geq c_{0}$, a sufficient condition (which is minimal) for the requirement to each single characteristic can be obtained by the following. Let $c^{\prime}$ be the minimum $S_{p k}$ value required for each single characteristic, then

$$
\frac{1}{3} \Phi^{-1}\left\{\left[\prod_{j=1}^{v}\left(2 \Phi\left(3 S_{p k j}\right)-1\right)+1\right] / 2\right\} \geq \frac{1}{3} \Phi^{-1}\left\{\left[\prod_{j=1}^{v}\left(2 \Phi\left(3 c^{\prime}\right)-1\right)+1\right] / 2\right\}
$$

Hence, if

$$
\frac{1}{3} \Phi^{-1}\left\{\left[\prod_{j=1}^{v}\left(2 \Phi\left(3 c^{\prime}\right)-1\right)+1\right] / 2\right\} \geq c_{0}
$$

i.e.

$$
c^{\prime} \geq \frac{1}{3} \Phi^{-1}\left(\frac{\sqrt[v]{2 \Phi\left(3 c_{0}\right)-1}+1}{2}\right)
$$

then we have

$$
S_{p k}^{\mathrm{T}}=\frac{1}{3} \Phi^{-1}\left\{\left[\prod_{j=1}^{v}\left(2 \Phi\left(3 S_{p k j}\right)-1\right)+1\right] / 2\right\} \geq c_{0}
$$


Table II. Capability zones for multiple characteristics

\begin{tabular}{ccc}
\hline & \multicolumn{2}{c}{$S_{p k j}$ for single characteristic } \\
\cline { 2 - 3 } Characteristic \\
number $v$ & Lower bound $s_{L}$ & Upper bound $s_{U}$ \\
\hline 1 & 1.000 & 1.333 \\
2 & 1.068 & 1.387 \\
3 & 1.107 & 1.417 \\
4 & 1.133 & 1.439 \\
5 & 1.153 & 1.455 \\
6 & 1.170 & 1.468 \\
7 & 1.183 & 1.479 \\
8 & 1.195 & 1.489 \\
9 & 1.205 & 1.497 \\
10 & 1.214 & 1.505 \\
11 & 1.222 & 1.511 \\
12 & 1.230 & 1.518 \\
13 & 1.236 & 1.523 \\
14 & 1.243 & 1.528 \\
15 & 1.248 & 1.533 \\
\hline
\end{tabular}

Thus, if the requirement for each single characteristic

$$
S_{p k j} \geq \frac{1}{3} \Phi^{-1}\left(\frac{\sqrt[v]{2 \Phi\left(3 c_{0}\right)-1}+1}{2}\right), \quad \text { for all } j=1,2, \ldots, v
$$

is satisfied, then the overall capability requirement $S_{p k}^{\mathrm{T}} \geq c_{0}$ would be satisfied. For example, if $c_{0}$ is set to be 1.00 with $v=5$, i.e. the overall process yield is set to be no less than 0.9973 . The overall capability requirement $S_{p k}^{\mathrm{T}} \geq 1.00$ would be satisfied if each single characteristic yield is no less than $(0.997300204)^{1 / 5}=$ 0.99945950 (equivalent to $540 \mathrm{ppm}$ of non-conformity items), and the capability for all the five characteristics is

$$
S_{p k j} \geq \frac{1}{3} \Phi^{-1}\left(\frac{\sqrt[5]{2 \Phi(3)-1}+1}{2}\right)=1.153, \quad \text { for } j=1,2, \ldots, 5
$$

If the requirement of the overall process capability is $c_{1} \leq S_{p k}^{\mathrm{T}} \leq c_{2}$ for a process with $v$ characteristics. The requirement would be satisfied, if the capability of $j$ th characteristic satisfies $s_{L} \leq S_{p k j} \leq s_{U}$ for all $j=1,2, \ldots, v$, where the lower bound $s_{L}$ and the upper bound $s_{U}$ on each $S_{p k j}$ can be calculated, respectively, as

$$
s_{L}=\frac{1}{3} \Phi^{-1}\left(\frac{\sqrt[v]{2 \Phi\left(3 c_{1}\right)-1}+1}{2}\right) \quad \text { and } \quad s_{U}=\frac{1}{3} \Phi^{-1}\left(\frac{\sqrt[v]{2 \Phi\left(3 c_{2}\right)-1}+1}{2}\right)
$$

Table II displays the lower bound $s_{L}$ and upper bound $s_{U}$ on $S_{p k j}$ if the requirement of the overall process capability is $1.000 \leq S_{p k}^{\mathrm{T}} \leq 1.333$ for $v=1(1) 15$ characteristics. For example, suppose the requirement of the overall process capability is $1.000 \leq S_{p k}^{\mathrm{T}} \leq 1.333$ for process with five characteristics $(v=5)$, we can obtain the lower bound $s_{L}=1.153$ and the upper bound $s_{U}=1.455$ on all the five $S_{p k j}$ values.

\section{S $S_{p k}$ MULTI-CHARACTERISTIC PROCESS CAPABILITY ANALYSIS CONTROL CHART}

Based on the yield index $S_{p k}$, Boyles ${ }^{4}$ developed a tool called the $S_{p k}$ contour plot which is a contour plot of index $S_{p k}$ as a function of the process parameters $(\mu, \sigma)$ for monitoring and controlling process performance. 


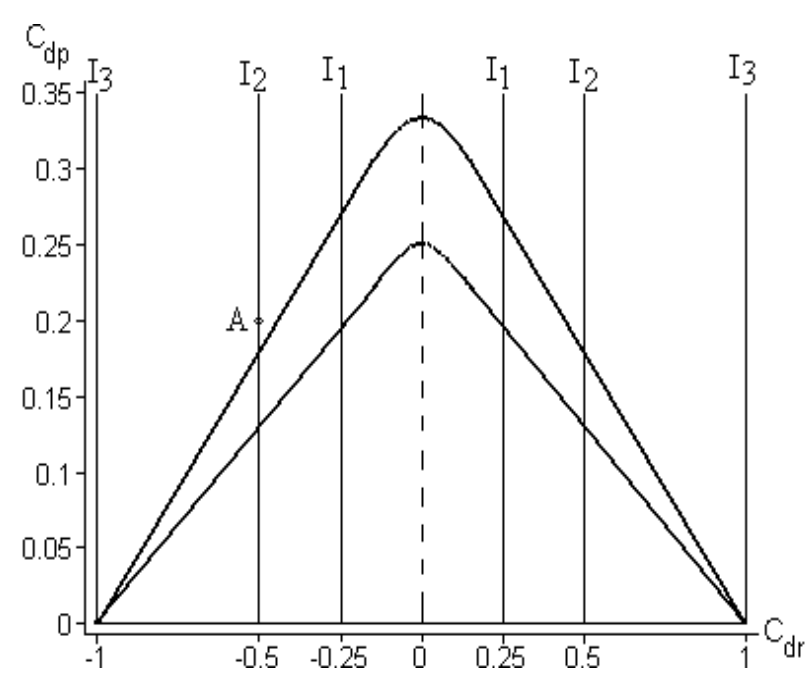

Figure 1. The $S_{p k}$ MCPCA control chart with various departure control zones

In fact, the $S_{p k}$ contour plot is a useful tool for evaluating multiple processes, as we can obtain the process yield and the process departure ratio by checking the location of the index value falling on the contour plot. For multiple processes with the same specification limits, the contour plot not only shows the process capability for multiple processes simultaneously, but also provides a quick reference to the parameters that should be targeted for process improvement. Note, however, that the $S_{p k}$ contour plot is only applicable for multiple processes with the same specification limits on each single process, which may not be used on processes with multiple characteristics where the characteristic specifications are not the same.

To extend the applicability of the contour plot for processes with multiple characteristics, we apply the method developed by Deleryd and Vännman ${ }^{9}$ who introduced a process capability plot, called the $(\delta, \gamma)$-plot, which is an adjustment of Boyles' $(\mu, \sigma)$-plot where $\delta=(\mu-T) / d, \gamma=\sigma / d$. We rewrite the definition of $S_{p k}$ as below, which can be expressed as a function of $C_{d r}=(\mu-T) / d$ and $C_{d p}=\sigma / d$. Note that $C_{d r}$ measures the departure ratio, and $C_{d p}$ measures the variation relative to the specification tolerance. If $\mu<T$, we have $C_{d r}<0$; if $\mu>T$, we have $C_{d r}>0$; and if $\mu=T$, we have $C_{d r}=0$ (the process is on target in this case). Obviously, if $\mu=\mathrm{LSL}$, then $C_{d r}=-1$; if $\mu=\mathrm{USL}$, then $C_{d r}=1$. We will focus on the case where the specification interval is twosided with the target value $T$ at $m$, which is most common in practical situations. Henceforth we will assume that $T=m$.

$$
\begin{aligned}
S_{p k} & =\frac{1}{3} \Phi^{-1}\left\{\frac{1}{2} \Phi\left(\frac{\mathrm{USL}-\mu}{\sigma}\right)+\frac{1}{2} \Phi\left(\frac{\mu-\mathrm{LSL}}{\sigma}\right)\right\} \\
& =\frac{1}{3} \Phi^{-1}\left\{\frac{1}{2} \Phi\left(\frac{1-(\mu-m) / d}{\sigma / d}\right)+\frac{1}{2} \Phi\left(\frac{1+(\mu-m) / d}{\sigma / d}\right)\right\} \\
& =\frac{1}{3} \Phi^{-1}\left\{\frac{1}{2} \Phi\left(\frac{1-C_{d r}}{C_{d p}}\right)+\frac{1}{2} \Phi\left(\frac{1+C_{d r}}{C_{d p}}\right)\right\}
\end{aligned}
$$

Therefore, using $C_{d r}$ as the $x$-axis and $C_{d p}$ as the $y$-axis, we can plot the following point set forming the curve of $S_{p k}$ (bold curves in Figure 1$)$ on the $\left(C_{d r}, C_{d p}\right)$ coordinates,

$$
\left\{\left(C_{d r}, C_{d p}\right) \mid \frac{1}{3} \Phi^{-1}\left[\frac{1}{2} \Phi\left(\frac{1-C_{d r}}{C_{d p}}\right)+\frac{1}{2} \Phi\left(\frac{1+C_{d r}}{C_{d p}}\right)\right]=S_{p k}\right\}
$$

Note that the process capability plot is invariable irrespective of the value of the specification limits. Processes with multiple characteristics having different characteristic specification limits can thus be plotted 
Table III. Various control regions for process departure and improvement suggestions

\begin{tabular}{ccl}
\hline Control region & $C_{d r}$ value & Process improvement suggestion \\
\hline$I_{1}$ & \pm 0.25 & $\begin{array}{l}\text { Characteristic departure is tolerable } \\
\text { Characteristic departure is abnormal, } \\
I_{2}\end{array}$ \\
$I_{3}$ & \pm 1.50 & $\begin{array}{l}\text { need to investigate and improve } \\
\text { Characteristic departure is serious, } \\
\text { need to recheck overall process }\end{array}$ \\
\hline
\end{tabular}

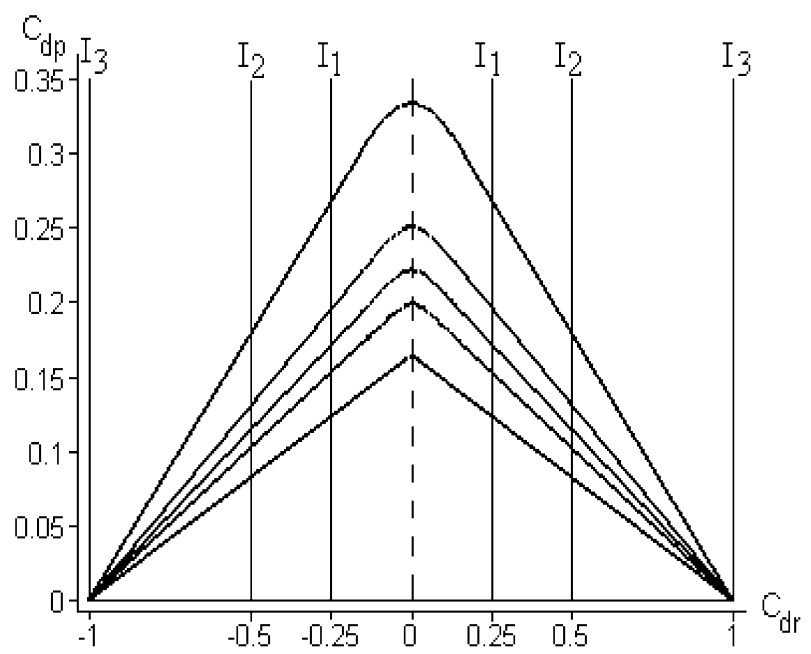

Figure 2. The MCPCA chart with contours of $S_{p k}=1.0,1.33,1.5,1.67,2.0$ (from top to bottom)

simultaneously on a single chart. We shall call this control chart the multi-characteristic process capability analysis (MCPCA) chart. In Figure 1, the top curve is the capability plot for processes with $S_{p k}=1.00$, and the bottom curve is the capability plot for processes with $S_{p k}=1.33$.

As we pointed out earlier, $S_{p k}$ is a yield-based index. However, according to today's modern quality improvement theories, reduction of variation from the target is as important as increasing the process yield (meeting the specifications). Therefore, three pairs of control limits $I_{1}, I_{2}$, and $I_{3}$ are drawn on the proposed MCPCA chart (see Figure 1) to monitor the variation from the target of each characteristic. Corresponding to the three pairs of control limits $I_{1}, I_{2}$, and $I_{3}$, the values of $C_{d r}=(\mu-T) / d$ are $-1.0,-0.5,-0.25,0.25,0.5$, and 1.0 from left to right, respectively.

Under the six-sigma quality improvement program formulated by Motorola (see Noguera and Nielsen ${ }^{12}$ ) assuming $d=6 \sigma$, the three pairs of control limits $I_{1}, I_{2}$, and $I_{3}$ correspond to $|\mu-T|=1.5 \sigma, 3 \sigma$ and $6 \sigma$. Research has shown that a typical process is likely to deviate from its natural centering condition by approximately $1.5 \sigma$ at any given moment in time. Under the six-sigma quality improvement program, the process mean is allowed to shift as much as $1.5 \sigma$, i.e. all the pairs $\left(C_{d r j}, C_{d p j}\right)$ of the $j$ th characteristic should not locate outside of the pair of control limits $I_{1}$. Note that six-sigma technically means having no more than $3.4 \mathrm{ppm}$ of non-conformities by assuming that the specification limits are $6 \sigma$ away from the target. Therefore, the three pairs of control limits $I_{1}, I_{2}$, and $I_{3}$ form various process accuracy (the degree of centering) control zones.

Various control regions (zones) for process departure and the improvement suggestions are summarized in Table III. The practitioners can judge the degree of centering of characteristic $j$ by checking the location of the corresponding plotted point on the MCPCA chart. For example, the departure measure $C_{d r}$ value is -0.5 for characteristic A in Figure 1. The departure ratio is considered to be significant which calls for an 
immediate check to find abnormal changes in the parameter settings. Using the MCPCA chart, the practitioners can effectively control and monitor both the variation and the departure from its target for each single process characteristic.

In the MCPCA chart (see Figure 2), we use the standardized measures on process departure and process variation. Therefore, the MCPCA chart can be used for processes with multiple characteristics where the individual characteristic specifications may not be identical. The MCPCA chart displays all the characteristic index values on one chart, and indicates the characteristic yield based on the $S_{p k}$ contours. It can provide information instantly about the locations and spreads of all the studied characteristics by their $\left(C_{d r}, C_{d p}\right)$-coordinate values. The MCPCA chart also displays the relative magnitudes of process variation and the process departure in terms of the standardized measures $\left(C_{d r}, C_{d p}\right)$ which can be used to control and monitor all characteristics simultaneously. Therefore, the MCPCA chart provides in-time information so that the practitioners can understand the quality level of the product easily. In addition, the MCPCA chart also provides a clear direction on which parameter needs to be targeted for quality improvement.

\section{AN APPLICATION}

Development of the new index is novel and deals with a common and practical problem (yield measurement) occurring in the manufacturing industry. To the best of our knowledge, none of the existing methods published in the literature provides the same function as the new index $S_{p k}^{\mathrm{T}}$. The new index is useful to the engineers/practitioners in measuring the yield, particularly for processes with multiple characteristics (a problem the industry must continue to face). We note that the exact sampling distribution of the estimated $S_{p k}$, which is a special case of the proposed new index with $v=1$ (for single characteristic), has never been investigated. Statistical properties of the special case with $v=1$ are in fact mathematically intractable. Lee et al. $^{13}$ obtained an approximate distribution of the estimated $S_{p k}$ using the Taylor expansion. Statistical properties of the new index, $S_{p k}^{\mathrm{T}}$, are expected to be considerably more difficult to deal with than that of $S_{p k}$ (for a single characteristic).

Pearn and Kang ${ }^{14}$ considered the sampling distribution of the estimated $S_{p k}$, and conducted a simulation to evaluate its accuracy. Their simulation results show that the estimated $S_{p k}$ overestimates the true value of $S_{p k}$. However, with a sample size $n$ greater than 150 , the relative bias is less than 0.01 , and the sampling error becomes negligible. For practical purposes, we suggest that a minimal value of $n=150$ must be used in the applications to maintain a good accuracy.

For each single characteristic the $C_{d r j}$ and $C_{d p j}$ values are calculated and the $\left(C_{d r j}, C_{d p j}\right)$ pair is plotted on the chart for $j=1,2, \ldots, v$. In real applications $C_{d r j}$ and $C_{d p j}$, representing the $j$ th characteristic (with target value $T_{j}$ ), are replaced by their natural estimators:

$$
\hat{C}_{d r j}=\frac{\left(\bar{X}_{j}-T_{j}\right)}{d_{j}}, \quad \hat{C}_{d p j}=\frac{s_{j}}{d_{j}}
$$

where $d_{j}$ is the half length of the $j$ th characteristic's specification interval, $\bar{X}_{j}=\sum_{i=1}^{n} x_{i j} / n$ and $s_{j}^{2}=$ $\sum_{i=1}^{n}\left(x_{i j}-\bar{X}_{j}\right)^{2} /(n-1)$ are the sample mean and the sample variance of the $j$ th characteristic, respectively. Hence, the natural estimators $\hat{S}_{p k j}$ of index $\mathrm{S}_{p k j}$ corresponding to the $j$ th characteristic are

$$
\hat{S}_{p k j}=\frac{1}{3} \Phi^{-1}\left\{\frac{1}{2} \Phi\left(\frac{1-\hat{C}_{d r j}}{\hat{C}_{d p j}}\right)+\frac{1}{2} \Phi\left(\frac{1+\hat{C}_{d r j}}{\hat{C}_{d p j}}\right)\right\}, \quad \text { for } j=1,2, \ldots, v
$$

To illustrate the application of the MCPCA chart, we consider a real example taken from an electronic thermos manufacturer, located in Taiwan, adapting the six-sigma quality improvement program. One special type of thermos investigated has five target-the-best quality characteristics with unequal manufacturing specifications.

The quality requirement for the final product is $1.00 \leq S_{p k}^{\mathrm{T}} \leq 1.33$, i.e. the requirement for the process yield is no less than $99.73 \%$. For $v=5$, we can obtain the lower bound $s_{L}=1.153$ and the upper bound $s_{U}=1.455$ for 
Table IV. Calculations for the electronic thermos process capability

\begin{tabular}{cccccccc}
\hline$j$ & LSL & $T$ & USL & $\bar{X}_{j}$ & $s_{j}$ & $\left(\hat{C}_{d r j}, \hat{C}_{d p}\right)$ & $\hat{S}_{p k j}$ \\
\hline 1 & 5.598 & 6.220 & 6.842 & 5.909 & 0.124 & $(-0.500,0.199)$ & 0.915 \\
2 & 606.5 & 680.0 & 753.5 & 683.3 & 17.13 & $(-0.045,0.233)$ & 1.406 \\
3 & 0.279 & 0.310 & 0.341 & 0.332 & 0.0076 & $(0.710,0.245)$ & 0.521 \\
4 & 31.5 & 35.0 & 38.5 & 34.48 & 0.525 & $(-0.149,0.150)$ & 1.931 \\
5 & 30 & 40 & 50 & 43.5 & 0.80 & $(0.35,0.08)$ & 2.737 \\
\hline
\end{tabular}

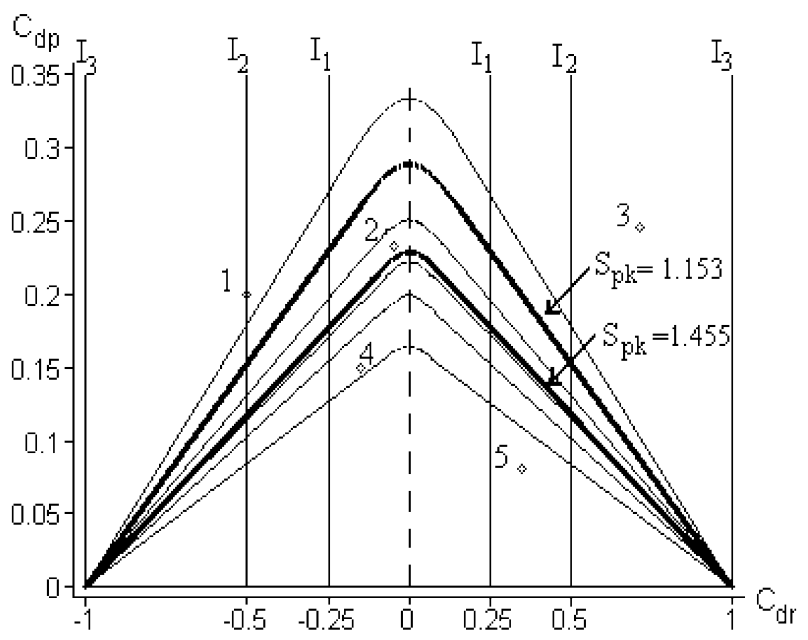

Figure 3. An application of the $S_{p k}$ MCPCA chart

all five $S_{p k j}$ values from Table II. Hence, a process is capable if $1.153 \leq S_{p k j} \leq 1.455$ and the pair $\left(C_{d r j}, C_{d p j}\right)$ of the $j$ th characteristic must locate between the pair control limits $I_{1}$ for all $j=1,2, \ldots, 5$.

A sample of 150 or more items is taken from the factory to inspect. The specification, target value, sample mean, sample standard deviation, estimated $C_{d r j}, C_{d p j}$, and $S_{p k j}$ are summarized in Table IV. First, we plot two contours corresponding to $S_{p k}=1.153$ and $S_{p k}=1.455$ on the MCPCA chart, shown as two bold curves in Figure 3. At the same time, five pairs of the estimated $C_{d r j}$ and $C_{d p j}$ values are plotted on the chart. This chart clearly shows the status of each in-process characteristic. Since all of these in-process characteristics are plotted in one chart, it is easy to determine their relative status. From the MCPCA chart, we can quickly conclude that the process is incapable because there are two plotted points out of the contour of $S_{p k}=s_{L}=1.153$. We note that the estimated $S_{p k}^{\mathrm{T}}=0.5135$. Based on the analysis of this chart, we can make the following conclusions and recommendations.

1. The plotted points corresponding to characteristics 1 and 3 are out of the contour of $S_{p k}=1.153$ which show both $\hat{S}_{p k 1}$ and $\hat{S}_{p k 3}$ are less than the lower bound value $s_{L}=1.153$ (the lower bound of quality requirement for all five $S_{p k j}$ ). Hence, the process is considered to be 'incapable' for characteristics 1 and 3. In fact, $\hat{S}_{p k 1}=0.915$ and $\hat{S}_{p k 3}=0.521$. Since both $\hat{S}_{p k 1}$ and $\hat{S}_{p k 3}$ are significantly less than 1.00 (the lower bound of quality requirement $S_{p k}^{\mathrm{T}}$ for the final product), then both characteristics 1 and 3 are candidates for high-priority quality improvement efforts. Furthermore, the plotted points 1 and 3 are out of the control limits $I_{1}$. Hence, both the variability and the deviation from target must be reduced to improve the process quality for characteristics 1 and 3 .

2. The plotted point corresponding to characteristic 2 is between two contours corresponding to $S_{p k}=1.153$ and $S_{p k}=1.455$. At the same time, the plotted point 2 is located between the pair of the control limits $I_{1}$. This shows that the process is satisfactory for characteristic 2 . Note that the corresponding $\hat{S}_{p k 2}=1.406$. 
3. The plotted points corresponding to characteristics 4 and 5 are inside the contour of $S_{p k}=1.455$, which shows both $\hat{S}_{p k 4}$ and $\hat{S}_{p k 5}$ are greater than the upper bound value $s_{U}=1.455$ (the upper bound of quality requirement for all five $S_{p k j}$ ). In fact, $\hat{S}_{p k 4}=1.931$ and $\hat{S}_{p k 5}=2.737$. Note that the plotted point 4 is located between the pair of the control limits $I_{1}$, but the plotted point 5 is out of the control limits $I_{1}$. A reduced sampling plan for characteristic 4 could be considered since the process is super. Under the six-sigma program, the quality improvement effort for characteristic 5 could be focused on the reduction of the process deviation from its target.

\section{CONCLUSIONS}

Process capability indices have been widely used in the manufacturing industry, providing numerical measures on process precision, process accuracy, and process performance. Capability measures for processes with a single characteristic has been investigated extensively. However, capability measures for processes with multiple characteristics are comparatively neglected. In this paper, we proposed a generalized capability measure, called $S_{p k}^{\mathrm{T}}$, based on the yield index $S_{p k}$ proposed by Boyles ${ }^{4}$, for processes with multiple characteristics. We established a relationship between the new measure and the process yield. We also developed a control chart MCPCA based on the proposed new measure, which displays all the characteristic measures in one single chart. Using the control chart MCPCA, the engineers can effectively monitor and control the performance of all process characteristics simultaneously.

\section{Acknowledgements}

The authors would like to thank the Editor, Professor Douglas Montgomery, for his helpful suggestions. The authors would also like to thank the anonymous referees for their careful reading of the paper and for helpful comments which improved the paper. This paper was supported in part by the National Science Council, Taiwan, under the contract NSC 90-2218-E-167-002.

\section{REFERENCES}

1. Kane VE. Process capability indices. Journal of Quality Technology 1986; 18(1):41-52.

2. Pearn WL, Kotz S, Johnson NL. Distributional and inferential properties of process capability indices. Journal of Quality Technology 1992; 24(4):216-231.

3. Pearn WL, Lin GH, Chen KS. Distributional and inferential properties of the process accuracy and process precision indices. Communications in Statistics: Theory and Methods 1998; 27(4):985-1000.

4. Boyles RA. Process capability with asymmetric tolerances. Communications in Statistics: Computation and Simulation 1994; 23(3):615-643.

5. Chan LK, Cheng SW, Spiring FA. A new measure of process capability C pm. Journal of Quality Technology 1998; 20(3):162-175.

6. Choi BC, Owen DB. A study of a new process capability index. Communications in Statistics: Theory and Methods 1990; 19(4):1231-1245.

7. Kotz S, Johnson NL. Process Capability Indices. Chapman \& Hall: London, 1993.

8. Vännman K. A unified approach to capability indices. Statistica Sinica 1995; 5:805-820.

9. Deleryd M, Vännman K. Process capability plots—a quality improvement tool. Quality and Reliability Engineering International 1999; 15:213-217.

10. Pearn WL, Lin GH. Estimating capability index $C_{p k}$ for processes with asymmetric tolerances. Communications in Statistics: Theory and Methods 2000; 29(11):2593-2604.

11. Bothe DR. A capability study for an entire product. ASQC Quality Congress Transactions 1992; 46:172-178.

12. Noguera J, Nielsen T. Implement six sigma for interconnect technology. ASQC Quality Congress Transactions 1992; $538-544$ 
13. Lee JC, Hung HN, Pearn WL, Kueng TL. On the distribution of the estimated process yield index $S_{p k}$. Quality and Reliability Engineering International 2002; 18(2):111-116.

14. Pearn WL, Kang HY. A simulation program for testing process performance based on the yield. Working Paper, National Chiao Tung University, Taiwan, 2002.

\section{Authors' biographies}

K. S. Chen is a Professor in the Department of Industrial Engineering and Management, National Chin-Yi Institute of Technology, Taichung, Taiwan. He received his MS degree in Statistics from Cheng Kung University, and his PhD degree in Quality Management from the National Chiao Tung University, Taiwan.

W. L. Pearn is a Professor of Operations Research and Quality Management at the Department of Industrial Engineering and Management, National Chiao Tung University, Taiwan. He received his MS degree in Statistics and his PhD degree in Operations Research from the University of Maryland, College Park, MD, U.S.A. He worked for AT\&T Bell Laboratories at Switch Network Control and Process Quality Centers before he joined the National Chiao Tung University.

P. C. Lin received his MS degree in Statistics from the National Chung Hsing University, and his PhD degree in Quality Management from the National Chiao Tung University, Taiwan. Currently, he is an Associate Professor at the General Eduction Center, National Chin-Yi Institute of Technology, Taichung, Taiwan. 